\title{
稳定同位素红外光谱技术测定 $\mathrm{CO}_{2}$ 同位素校正方法 的研究进展
}

\section{庞家平 1,2 温学发 $1,3^{*}$}

${ }^{1}$ 中国科学院地理科学与资源研究所生态系统网络观测与模拟重点实验室, 北京 $100101 ;{ }^{2}$ 中国科学院南京地理与湖泊研究所流域地理学重点实验室, 南京 $210008{ }^{3}$ 中国科学院大学资源与环境学院, 北京 100190

\begin{abstract}
摘 要 稳定同位素红外光谱(IRIS)技术克服了传统的大气 $\mathrm{CO}_{2}$ 气瓶采样-同位素质谱(IRMS)技术时间分辨率低且耗时费力的 缺点, 可以实现高时间分辨率和高精度的大气 $\mathrm{CO}_{2}$ 碳同位素组成 $\left(\delta^{13} \mathrm{C}\right)$ 和氧同位素组成 $\left(\delta^{18} \mathrm{O}\right)$ 的原位连续测定。基于IRIS技术 测量 $\mathrm{CO}_{2} \delta^{13} \mathrm{C}$ 和 $\delta^{18} \mathrm{O}$ 的误差来源主要包括 $\delta^{13} \mathrm{C}$ 和 $\delta^{18} \mathrm{O}$ 测量值对 $\mathrm{CO}_{2}$ 浓度变化的非线性响应(浓度依赖性)以及对环境条件变化 的敏感性导致的漂移(时间漂移)。如何有效地校正浓度依赖性和时间漂移导致的误差是IRIS仪器应用的前提。该综述阐述了 $\delta^{13} \mathrm{C}$ 和 $\delta^{18} \mathrm{O}$ 测量值的浓度依赖性产生的理论基础, 回顾了浓度依赖性的理论校正和经验方程校正方法和应用; 回顾了时间漂 移的校正原理、方法和应用; 概述了数据溯源至国际标准的原理、方法与应用现状。结合实际情况推荐利用 3 个或 3 个以上已 知 $\mathrm{CO}_{2}$ 浓度和 $\delta^{13} \mathrm{C} 、 \delta^{18} \mathrm{O}$ 真值的 $\mathrm{CO}_{2}$ 标准气体涵盖待测气体 $\mathrm{CO}_{2}$ 浓度的浓度依赖性校正, 设置适当的校正频率校正时间漂移并 进行数据溯源。指出应该加强不同仪器和校正方法的比对研究; 采用IRIS技术测定 $\mathrm{CH}_{4} 、 \mathrm{~N}_{2} \mathrm{O}$ 和 $\mathrm{H}_{2} \mathrm{O}$ 同位素组成也可以采取类 似的校正方法。
\end{abstract}

关键词＼cjkstart稳定同位素红外光谱；浓度依赖性；时间漂移；数据溯源；校正频率

庞家平, 温学发 (2018). 稳定同位素红外光谱技术测定 $\mathrm{CO}_{2}$ 同位素校正方法的研究进展. 植物生态学报, 42, 143-152. DOI: 10.17521/cjpe.2017.0206

\section{A review of the calibration methods for measuring the carbon and oxygen isotopes in $\mathrm{CO}_{2}$ based on isotope ratio infrared spectroscopy}

PANG Jia-Ping ${ }^{1,2}$ and WEN Xue-Fa ${ }^{1,3^{*}}$

${ }^{1}$ Key Laboratory of Ecosystem Network Observation and Modeling, Institute of Geographic Sciences and Natural Resources Research, Chinese Academy of Sciences, Beijing 100101, China; ${ }^{2}$ Key Laboratory of Watershed Geographic Sciences, Nanjing Institute of Geography and Limnology, Chinese Academy of Sciences, Nanjing 210008, China; and ${ }^{3}$ College of Resources and Environment, University of Chinese Academy of Sciences, Beijing 100190, China

\section{Abstract}

With the development of isotope ratio infrared spectroscopy (IRIS) technology, it is now possible for the in situ high temporal resolution and high precision measurement of carbon isotopic composition $\left(\delta^{13} \mathrm{C}\right)$ and oxygen isotopic composition $\left(\delta^{18} \mathrm{O}\right)$ of atmospheric $\mathrm{CO}_{2}$, which overcomes the low temporal resolution and labor intensive shortcoming of traditional isotope ratio mass spectrometry (IRMS). The dependence of $\delta^{13} \mathrm{C}$ and $\delta^{18} \mathrm{O}$ on $\mathrm{CO}_{2}$ concentration (termed as concentration dependence) and the drift due to sensitivity to changing environmental conditions (termed as instrumental drift) are the two main sources of error affecting the IRIS measurements. Therefore, it is important to obtain precise measurements by constructing a proper calibration strategy to solve the concentration dependence and instrumental drift. In this study, we briefly discussed the definition and related theoretical principle of concentration dependence, and elaborated the theoretical and empirical calibration methods of concentration dependence. Moreover, we introduced the calibration methods of instrumental drift, and reviewed the state of the art of calibration methods and its application of IRIS technology. Additionally, we briefly discussed the definition and method of data traceability to the international standard, and reviewed its application of IRIS technology. Finally, we recommend that concentration dependence is corrected by using three standards or above with known $\mathrm{CO}_{2}$ concentration and its $\delta^{13} \mathrm{C}$ and $\delta^{18} \mathrm{O}$, bracketing the $\mathrm{CO}_{2}$ concentration of samples. The instrumental drift is corrected by setting appropriate calibration frequency and all dataset are traceable to the

收稿日期Received: 2017-08-09 接受日期Accepted: 2017-12-13

基金项目：国家重点研发计划(2016YFC0500102)和国家自然科学基金(31470500和41671257)。Supported by the National Key Research and Development Project of China (2016YFC0500102) and the National Natural Science Foundation of China (31470500 and 41671257).

* 通信作者Corresponding author (wenxf@igsnrr.ac.cn) 
international standard. In the future, the comparative study of different IRIS instruments and calibration methods should be enhanced, and the similar methods should be used for measuring $\mathrm{CH}_{4}, \mathrm{~N}_{2} \mathrm{O}$ and $\mathrm{H}_{2} \mathrm{O}$ isotopes by IRIS technique. The IRIS technology combined with other technology will provide a new opportunity for ecological research.

Key words isotope ratio infrared spectroscopy; concentration dependence; instrumental drift; traceability to the international standard; calibration frequency

Pang JP, Wen XF (2018). A review of the calibration methods for measuring the carbon and oxygen isotopes in $\mathrm{CO}_{2}$ based on isotope ratio infrared spectroscopy. Chinese Journal of Plant Ecology, 42, 143-152. DOI: 10.17521/cjpe.2017.0206

稳定同位素观测仪器和方法的进步使稳定同位 素技术成为解决生态、大气和水文等研究热点与难 点问题的重要工具(Yakir \& Sternberg, 2000; Bowling et al., 2003; Griffis, 2013; 林光辉, 2013; Chen et al., 2017)。稳定同位素红外光谱(IRIS)技术的发展使得 大气 $\mathrm{CO}_{2}$ 的碳同位素组成 $\left(\delta^{13} \mathrm{C}\right)$ 和氧同位素组成 $\left(\delta^{18} \mathrm{O}\right)$ 的高时间分辨率和高精度的原位连续观测成 为可能(McManus et al., 2010; Tanaka et al., 2013; Wen et al., 2013)。商业化的仪器包括调制式半导体 激光吸收光谱(TDLAS)、波长扫描光腔衰荡光谱 (WS-CRDS)、离轴综合腔输出光谱(OA-ICOS)、量 子级联激光吸收光谱 (QCLAS) 和差频激光光谱 (DFG)等(Griffis, 2013; Wen et al., 2013)。

IRIS技术突破了传统大气 $\mathrm{CO}_{2}$ 气瓶采样-稳定同 位素质谱(IRMS)技术的分辨率低且费时耗力的局 限性, 能够实现 $\mathrm{CO}_{2}$ 碳氧同位素比值的原位连续观 测(Bowling et al., 2003, 2005; Schaeffer et al., 2008; Bickford et al., 2010; Wingate et al., 2010; Shim et al., 2011)。所有IRIS仪器的观测结果都需要能够高精度 地测定自然条件下大气 $\mathrm{CO}_{2} \delta^{13} \mathrm{C}$ 和 $\delta^{18} \mathrm{O}$ 的变异特征, 并准确地追溯到国际标准(Bowling et al., 2005; Schaeffer et al., 2008; Griffis, 2013)。基于IRIS技术测 量 $\mathrm{CO}_{2} \delta^{13} \mathrm{C}$ 和 $\delta^{18} \mathrm{O}$ 的主要误差来源包括 $\delta^{13} \mathrm{C}$ 和 $\delta^{18} \mathrm{O}$ 测量值对 $\mathrm{CO}_{2}$ 浓度变化的非线性响应(浓度依赖性) 以及对环境条件变化的敏感性导致的漂移(时间漂 移)(Griffith et al., 2012; Guillon et al., 2012; Vogel et al., 2013)。例如, Guillon等(2015)研究表明, 在 300-17 $800 \mu \mathrm{mol} \cdot \mathrm{mol}^{-1} \mathrm{CO}_{2}$ 浓度范围内, 利用OAICOS 技术的Los Gatos Research CCIA-EP测定的 $\mathrm{CO}_{2} \delta^{13} \mathrm{C}$ 浓 度依赖性达到 $19 \%$, 而在300-1 $000 \mu \mathrm{mol} \cdot \mathrm{mol}^{-1}$ 的浓 度范围内达到 6\%o。Pang等(2016a)研究表明, 在 368.1-550.1 $\mu \mathrm{mol} \cdot \mathrm{mol}^{-1}$ 浓度范围内, 利用WS-CRDS 技术的Picarro G1101-i和G2201-i测定的 $\mathrm{CO}_{2} \delta^{13} \mathrm{C}$ 浓 度依赖性分别为 $1.75 \%$ 和 $0.47 \%$ 。仪器的长期漂移也
是影响IRIS仪器测量结果准确性的重要因素(Vogel et al., 2013; Guillon et al., 2015)。例如, Vogel等(2013) 研究表明, 利用WS-CRDS技术的Picarro G1101-i测 定的 $\mathrm{CO}_{2} \delta^{13} \mathrm{C}$ 在 $24 \mathrm{~h}$ 的漂移为 $0.3 \%$ 。Guillon等(2015) 利用OA-ICOS 技术的Los Gatos Research CCIA-EP 测定的 $\mathrm{CO}_{2} \delta^{13} \mathrm{C}$ 在 $24 \mathrm{~h}$ 内变化为 $5 \%$ 。 $\mathrm{Pang}$ 等(2016a) 在 $48 \mathrm{~h}$ 的测量周期内, 利用WS-CRDS技术的Picarro $\mathrm{G} 1101-\mathrm{i}$ 和 $\mathrm{G} 2201-\mathrm{i}$ 测定的 $\mathrm{CO}_{2} \delta^{13} \mathrm{C}$ 时间漂移分别为 $0.92 \%{ }_{0}-1.09 \%$ 和 $0.19 \%{ }_{0}-0.37 \%$ 。

采用适当的浓度依赖性和时间漂移的校正方法 是提升仪器的测量精度, 并获得可追溯到国际标准 的准确测量结果的重要保证。为了确保所有IRIS仪 器都能高精度地测定自然条件下 $\delta$ 值的变异特征, 并准确地溯源到国际标准, 需要对IRIS仪器的测量 结果进行适当的校正, 以消除浓度依赖性和时间漂 移带来的误差(Bowling et al., 2005; Schaeffer et al., 2008; Griffis, 2013)。目前已有的研究通常根据仪器 性能和研究需要采用不同的理论或经验的校正方法 进行浓度依赖性和时间漂移校正(Bowling et al., 2003; Tuzson et al., 2008; Vogel et al., 2013; Guillon et al., 2012, 2015)。尽管IRIS仪器在我国生态、大气 和水文等研究中得到广泛应用, 但还缺乏IRIS技术 的浓度依赖性和时间漂移等产生的原因及其校正方 法的系统论述。

本文概述了稳定同位素比值和同位素通量比值 的概念, 系统阐述了浓度依赖性的概念、校正原理、 理论校正及经验校正的方法和应用，阐述了时间漂 移的概念、校正方法和应用, 综述了数据溯源至国 际标准的原理、方法和应用, 推荐了浓度依赖性和 时间漂移等的校正方法, 并指出了IRIS技术和方法 应用研究的瓶颈问题和在生态学中的应用潜力。

\section{1 同位素比值与同位素通量比值}

稳定同位素是指不发生或极不易发生放射性衰 
变的具有相同质子数、不同中子数的同一元素的不 同核素。稳定同位素组成可以用同位素丰度或原子 百分比、绝对丰度、原子百分超或同位素比率表示 (林光辉, 2013)。由于重同位素的自然丰度较低, $\mathrm{CO}_{2}$ 的碳氧同位素组成通常用同位素比率 $(R)$ 来表示:

$$
\begin{aligned}
& { }^{13} R\left(\mathrm{CO}_{2}\right)=\frac{{ }^{13} \mathrm{C}}{{ }^{12} \mathrm{C}}=\frac{{ }^{13} \mathrm{CO}_{2}}{{ }^{12} \mathrm{CO}_{2}} \\
& { }^{18} R\left(\mathrm{CO}_{2}\right)=\frac{{ }^{18} \mathrm{O}}{{ }^{16} \mathrm{O}}=\frac{\mathrm{C}^{16} \mathrm{O}^{18} \mathrm{O}}{2 \times \mathrm{CO}_{2}}
\end{aligned}
$$

为便于比较, $\mathrm{CO}_{2}$ 碳氧同位素组成通常用同位 素比值 $(\delta$ 值)来表示, 其定义为:

$$
\delta_{\text {sample }}=\left(\frac{R_{\text {sample }}}{R_{\mathrm{VPDB}}}-1\right) \times 1000
$$

式中 $R_{\text {sample }}$ 是所测定样品的同位素比率, $R_{\mathrm{VPDB}}$ 是 $\mathrm{CO}_{2}$ 中碳或氧稳定同位素国际标准的同位素比率, 分别为 0.0111797 和 0.002088349077 。 $\delta_{\text {sample }}$ 表示 样品的同位素比率相对于标准物质同位素比率的相 对千分差, 可以清晰地反映同位素组成的变化。 $\delta$ 值 通常用千分数(\%)表示。

$\mathrm{CO}_{2}$ 通量的碳氧同位素组成通常用同位素通量 比率 $\left(R_{\mathrm{F}}\right)$ 来表示:

$$
\begin{aligned}
& { }^{13} R\left(\mathrm{CO}_{2}\right)_{\mathrm{F}}=\frac{F^{13} \mathrm{C}}{F^{12} \mathrm{C}}=\frac{F^{13} \mathrm{CO}_{2}}{F^{12} \mathrm{CO}_{2}} \\
& { }^{18} R\left(\mathrm{CO}_{2}\right)_{\mathrm{F}}=\frac{F^{18} \mathrm{O}}{F^{16} \mathrm{O}}=\frac{F \mathrm{C}^{16} \mathrm{O}^{18} \mathrm{O}}{2 \times F \mathrm{C}^{16} \mathrm{O}_{2}}
\end{aligned}
$$

其中, $F^{12} \mathrm{C}$ 和 $F^{13} \mathrm{C}\left(F^{16} \mathrm{O}\right.$ 和 $\left.F^{18} \mathrm{O}\right)$ 分别为轻重同位素 的通量。同位素通量比率可以由涡度协方差或者通 量廓线技术的进行观测和计算(Santos et al., 2012; Sturm et al., 2012; Wehr et al., 2016)。同样, 为便于 比较, $\mathrm{CO}_{2}$ 通量碳氧同位素组成也通常用同位素通 量比值 $(\delta$ 值)来表示, 其定义为:

$$
\delta_{F}=\left(\frac{R_{F}}{R_{\mathrm{VPDB}}}-1\right) \times 1000
$$

式中 $R_{F}$ 是所测定通量的同位素通量比率, $\delta$ 值通常用 千分数 $(\%)$ 表示。

$\mathrm{CO}_{2}$ 浓度是标量, 而 $\mathrm{CO}_{2}$ 通量是矢量, 同样同位 素比值 $(\delta$ 值) 是标量而同位素通量比值 $(\delta$ 值) 是矢量。

通常, IRIS仪器校正分为内部校正和外部校正。 内部校正是指试验前由仪器制造商或者仪器使用者 通过直接改变分析仪本身参数设置而达到校正目的 (Guillon et al., 2012; Sturm et al., 2012)。外部校正则
是指试验过程中仪器使用者通过设置不同的标准气 体来校正仪器浓度依赖性和时间漂移(Wen et al., 2013)。在本文中, 所指的 $\mathrm{CO}_{2}$ 碳氧同位素校正方法 都是针对同位素比值 $(\delta$ 值)的外部校正方法。

\section{2 浓度依赖性的校正原理、方法和应用}

\section{1 浓度依赖性产生的理论基础}

浓度依赖性是指 IRIS仪器测量具有相同 $\delta^{13} \mathrm{C}$ (或 $\delta^{18} \mathrm{O}$ ) 的不同浓度的 $\mathrm{CO}_{2}$ 标准气体时表现出的 $\delta$ 测 量值与 $\mathrm{CO}_{2}$ 浓度的非线性关系(Wen et al., 2013)。在 理想条件下, IRIS仪器应该没有浓度依赖性现象, 即具有相同 $\delta^{13} \mathrm{C}\left(\right.$ 或 $\delta^{18} \mathrm{O}$ )值的不同浓度 $\mathrm{CO}_{2}$ 的 $\delta$ 测量 值应该恒定不变。由于IRIS仪器直接测量每个核素 的浓度, 根据测量温度和压力等将浓度转换为摩尔 混合比率后计算 $\delta$ 值(Wehr et al., 2013), 因此, 单个 核素浓度的测量值与真值之间的测量误差导致了 IRIS仪器的浓度依赖性(Griffith et al., 2012)。

IRIS仪器 $\delta$ 测量值对浓度变化的浓度依赖性是 测量误差的主要来源。以 $\mathrm{CO}_{2} \delta^{13} \mathrm{C}$ 为例, 当仪器对 ${ }^{12} \mathrm{CO}_{2}$ 和 ${ }^{13} \mathrm{CO}_{2}$ 浓度的响应完全线性时, 由于仪器的 测量误差, ${ }^{12} \mathrm{CO}_{2}$ 和 ${ }^{13} \mathrm{CO}_{2}$ 测量值与真值之间会存在 微小的差异, 可以通过线性回归方程进行计算。

$$
\begin{aligned}
& C_{12, \mathrm{~T}}=a_{12} \times C_{12, \mathrm{M}}+b_{12} \\
& C_{13, \mathrm{~T}}=a_{13} \times C_{13, \mathrm{M}}+b_{13}
\end{aligned}
$$

式中 $C$ 为 $\mathrm{CO}_{2}$ 的浓度, 下标 $12 、 13$ 分别代表 ${ }^{12} \mathrm{CO}_{2}$ 和 ${ }^{13} \mathrm{CO}_{2}$, T和 $\mathrm{M}$ 分别为真值和测量值; $a_{12} 、 b_{12}$ 和 $a_{13} 、 b_{13}$ 分别为 ${ }^{12} \mathrm{CO}_{2}$ 和 ${ }^{13} \mathrm{CO}_{2}$ 的校正系数。如果不对 ${ }^{12} \mathrm{CO}_{2}$ 和 ${ }^{13} \mathrm{CO}_{2}$ 进行校正直接计算 $\delta$ 值, 那么 $\delta$ 值可以表示为:

$$
\delta_{\mathrm{T}}=\frac{a_{13} C_{12}}{a_{12} C_{12}+b_{12}} \delta_{\mathrm{M}}+\frac{\left(a_{13}-a_{12}\right) C_{12}+b_{13}-b_{12}}{a_{12} C_{12}+b_{12}}
$$

根据上述方程可知, 如果 $b_{12}$ 和 $b_{13}$ 不为 0 , 那么 方程中的斜率和截距将会随着 $\mathrm{CO}_{2}$ 浓度的变化而变 化, 从而导致 $\delta$ 值对 $\mathrm{CO}_{2}$ 浓度的依赖性, 并且在一定 $\mathrm{CO}_{2}$ 浓度范围内表现出非线性响应。因此, 当仪器对 ${ }^{12} \mathrm{CO}_{2}$ 和 ${ }^{13} \mathrm{CO}_{2}$ 浓度的响应为非完全线性时, 也必然 会导致 $\delta$ 值的浓度依赖性(Wen et al., 2013)。

根据上述方程，即使浓度依赖性的响应为非线 性的, 在较小的浓度范围内也可近似地认为 $\delta$ 值的 浓度依赖性是线性的。例如, Pang等(2016a)研究表 明在368.1-550.1 $\mu \mathrm{mol} \cdot \mathrm{mol}^{-1}$ 浓度范围内基于WSCRDS技术的 G1101-i和 G2201-i的 $\delta^{13} \mathrm{C}$ 浓度依赖性 分别为每 $100 \mu \mathrm{mol} \cdot \mathrm{mol}^{-1} 0.46 \%$ 和每 $100 \mu \mathrm{mol} \cdot \mathrm{mol}^{-1}$ 
0.09\% 。 Guillon等(2012)利用基于OA-ICOS技术的 Los Gatos Research DLT-100碳同位素分析仪分别测 定1 $920 \mu \mathrm{mol} \cdot \mathrm{mol}^{-1}(-43.99 \%)$ 和17 $800 \mu \mathrm{mol} \cdot \mathrm{mol}^{-1}$ $(-39.37 \% 0)$ 标准气体的逐级稀释气体, 结果表明在 300-2 $000 \mu \mathrm{mol} \cdot \mathrm{mol}^{-1}$ 存在非线性的 $\delta^{13} \mathrm{C}$ 浓度依赖性, 2 个标准气体在1 $000-2000 \mu \mathrm{mol} \cdot \mathrm{mol}^{-1}$ 范围内的浓 度依赖性拟合曲线重叠得很好, 这表明在- $44 \%$ 。到 $-39 \%$ 范围内 $\delta^{13} \mathrm{C}$ 的浓度依赖性不受 $\mathrm{CO}_{2}$ 同位素组 成变化的影响。

理想的校正方法是利用不同 $\delta$ 值的 $\mathrm{CO}_{2}$ 标准气 体或固体物质产生多个浓度梯度系列涵盖待测气体 $\mathrm{CO}_{2}$ 浓度和 $\delta$ 值的变化范围, 可有效校正仪器的浓度 依赖性。通常 $\mathrm{CO}_{2}$ 标准气体浓度涵盖待测气体 $\mathrm{CO}_{2}$ 浓度的范围比其涵盖 $\delta$ 值的范围更为重要(Bowling et al., 2003; Griffis et al., 2005; Wen et al., 2013)。根 据浓度依赖性的定义和产生原因, 可以将校正方法 分为理论校正和经验校正方法。

\section{2 浓度依赖性的理论校正方法与应用}

IRIS 仪器可以直接测定 ${ }^{12} \mathrm{CO}_{2}$ 和 ${ }^{13} \mathrm{CO}_{2}$ 或 $\mathrm{C}^{16} \mathrm{O}_{2}$ 和 $\mathrm{C}^{18} \mathrm{O}^{16} \mathrm{O}$ 浓度, 根据浓度依赖性产生的理论原因, 只要分别准确校正 ${ }^{12} \mathrm{CO}_{2}$ 和 ${ }^{13} \mathrm{CO}_{2}$ 或 $\mathrm{C}^{16} \mathrm{O}_{2}$ 和 $\mathrm{C}^{18} \mathrm{O}^{16} \mathrm{O}$ 浓度就可以直接校正仪器的浓度依赖性。因此, 首 先需要获得 $\mathrm{CO}_{2}$ 标准气体的 ${ }^{12} \mathrm{CO}_{2}$ 和 ${ }^{13} \mathrm{CO}_{2}$ 或 $\mathrm{C}^{16} \mathrm{O}_{2}$ 和 $\mathrm{C}^{18} \mathrm{O}^{16} \mathrm{O}$ 浓度。

对 $\mathrm{CO}_{2}$ 标准气体来说, 从已知总 $\left[\mathrm{CO}_{2}\right]$ 和 $\delta^{13} \mathrm{C}$ 真 值可以获得 $\mathrm{CO}_{2}$ 标准气体中的 $\left[{ }^{12} \mathrm{CO}_{2}\right]$ 和 $\left[{ }^{13} \mathrm{CO}_{2}\right]$, 计 算公式如下:

$$
\begin{aligned}
& {\left[\mathrm{CO}_{2}\right]=\left[{ }^{12} \mathrm{CO}_{2}\right]+\left[{ }^{13} \mathrm{CO}_{2}\right]+f\left[\mathrm{CO}_{2}\right]} \\
& R_{\mathrm{a}}=\left[{ }^{13} \mathrm{C}\right] /\left[{ }^{12} \mathrm{C}\right]=\left[{ }^{13} \mathrm{CO}_{2}\right] /\left[{ }^{13} \mathrm{CO}_{2}\right] \\
& \quad=R_{\mathrm{VPDB}}\left(1+\delta_{\mathrm{a}} / 1000\right) \\
& {\left[{ }^{12} \mathrm{CO}_{2}\right]=\left[\mathrm{CO}_{2}\right](1-f) /\left(1+R_{\mathrm{VPDB}}\left(1+\delta_{\mathrm{a}} / 1000\right)\right)} \\
& {\left[{ }^{13} \mathrm{CO}_{2}\right]=\left[\mathrm{CO}_{2}\right](1-f)-\left[{ }^{12} \mathrm{CO}_{2}\right]}
\end{aligned}
$$

其中 $\left[\mathrm{CO}_{2}\right]$ 是包括所有同位素的总 $\mathrm{CO}_{2}$ 浓度; $f$ 是除了 $\left[{ }^{12} \mathrm{CO}_{2}\right]$ 和 $\left[{ }^{13} \mathrm{CO}_{2}\right]$ 外其他同位素含量的比例(0.004 74); $R_{\mathrm{a}}$ 是 $\mathrm{CO}_{2}$ 标准气体已知 ${ }^{13} \mathrm{C} /{ }^{12} \mathrm{C} ; R_{\mathrm{VPDB}}$ 是国际标准 (VPDB)的 ${ }^{13} \mathrm{C} /{ }^{12} \mathrm{C}$ 同位素比率(0.011 179 7); $\delta_{\mathrm{a}}$ 是 $\mathrm{CO}_{2}$ 标准气体已知 $\delta^{13} \mathrm{C}$ 。

同样, 从已知总 $\left[\mathrm{CO}_{2}\right]$ 和 $\delta^{18} \mathrm{O}$ 真值可以获得 $\mathrm{CO}_{2}$ 标准气体中的 $\left[\mathrm{C}^{16} \mathrm{O}_{2}\right]$ 和 $\left[\mathrm{C}^{18} \mathrm{O}^{16} \mathrm{O}\right]$, 计算公式如下:

$$
\left[\mathrm{CO}_{2}\right]=\left[\mathrm{C}^{16} \mathrm{O}_{2}\right]+\left[\mathrm{C}^{18} \mathrm{O}^{16} \mathrm{O}\right]+f\left[\mathrm{CO}_{2}\right]
$$

$$
\begin{aligned}
& R_{\mathrm{a}}=\left[{ }^{18} \mathrm{O}\right] /\left[{ }^{16} \mathrm{O}\right]=0.5 \times\left[\mathrm{C}^{18} \mathrm{O}^{16} \mathrm{O}\right] /\left[\mathrm{C}^{16} \mathrm{O}_{2}\right] \\
& =R_{\mathrm{VPDB}}\left(1+\delta_{\mathrm{a}} / 1000\right) \\
& {\left[\mathrm{C}^{16} \mathrm{O}_{2}\right]=\left[\mathrm{CO}_{2}\right](1-f) /\left(1+2 R_{\mathrm{VPDB}}\left(1+\delta_{\mathrm{a}} / 1000\right)\right)} \\
& {\left[\mathrm{C}^{18} \mathrm{O}^{16} \mathrm{O}\right]=\left[\mathrm{CO}_{2}\right](1-f)-\left[\mathrm{C}^{16} \mathrm{O}_{2}\right]}
\end{aligned}
$$

其中 $f$ 是除了 $\mathrm{C}^{16} \mathrm{O}_{2}$ 和 $\mathrm{C}^{18} \mathrm{O}^{16} \mathrm{O}$ 外其他同位素含量的 比例( 0.01185$) ; R_{\mathrm{a}}$ 是 $\mathrm{CO}_{2}$ 标准气体已知 ${ }^{18} \mathrm{O} /{ }^{16} \mathrm{O}, 0.5$ 是 $\mathrm{C}^{18} \mathrm{O}^{16} \mathrm{O}$ 中存在轻重2个氧原子的比例系数; $R_{\mathrm{VPDB}}$ 是国际标准的 ${ }^{18} \mathrm{O} /{ }^{16} \mathrm{O}$ 同位素比率(0.002 088349077 )。

通常, 至少需要 2 个或以上 $\mathrm{CO}_{2}$ 标准气体的 $\mathrm{CO}_{2}$ 浓度涵盖待测目标气体 $\mathrm{CO}_{2}$ 浓度, 同时根据上述公 式(10)-(13)计算所需 ${ }^{12} \mathrm{CO}_{2}$ 和 ${ }^{13} \mathrm{CO}_{2}$ 或公式(14)-(17) 计算所需 $\mathrm{C}^{16} \mathrm{O}_{2}$ 和 $\mathrm{C}^{18} \mathrm{O}^{16} \mathrm{O}$ 的浓度真值, 建立相应的 真值与测量值的函数关系对目标气体 ${ }^{12} \mathrm{CO}_{2}$ 和 ${ }^{13} \mathrm{CO}_{2}$ 或 $\mathrm{C}^{16} \mathrm{O}_{2}$ 和 $\mathrm{C}^{18} \mathrm{O}^{16} \mathrm{O}$ 进行校正。例如, 利用 2 个 $\mathrm{CO}_{2}$ 标 准气体可以分别建立轻重同位素 $\left({ }^{12} \mathrm{CO}_{2}\right.$ 和 ${ }^{13} \mathrm{CO}_{2}$ 或 $\mathrm{C}^{16} \mathrm{O}_{2}$ 和 $\mathrm{C}^{16} \mathrm{O}^{18} \mathrm{O}$ ) 浓度的真值和测量值的线性函数 关系并对目标气体进行校正，校正方程可以表示为:

$$
\begin{aligned}
& X_{\mathrm{s}, \mathrm{T}}^{\mathrm{L}}=\frac{X_{2, \mathrm{~T}}^{\mathrm{L}}-X_{1, \mathrm{~T}}^{\mathrm{L}}}{X_{2, \mathrm{M}}^{\mathrm{L}}-X_{1, \mathrm{M}}^{\mathrm{L}}}\left(X_{\mathrm{s}, \mathrm{M}}^{\mathrm{L}}-X_{1, \mathrm{M}}^{\mathrm{L}}\right)+X_{1, \mathrm{~T}}^{\mathrm{L}} \\
& X_{\mathrm{s}, \mathrm{T}}^{\mathrm{H}}=\frac{X_{2, \mathrm{~T}}^{\mathrm{H}}-X_{1, \mathrm{~T}}^{\mathrm{H}}}{X_{2, \mathrm{M}}^{\mathrm{H}}-X_{1, \mathrm{M}}^{\mathrm{H}}}\left(X_{\mathrm{s}, \mathrm{M}}^{\mathrm{H}}-X_{1, \mathrm{M}}^{\mathrm{H}}\right)+X_{1, \mathrm{M}}^{\mathrm{H}}
\end{aligned}
$$

其中 $X_{\mathrm{s}, \mathrm{T}} 、 X_{1, \mathrm{~T}}$ 和 $X_{2, \mathrm{~T}}$ 为目标气体、标准气体 1 和 2 的摩 尔浓度真值; $X_{\mathrm{s}, \mathrm{M}} 、 X_{1, \mathrm{M}}$ 和 $X_{2, \mathrm{M}}$ 为目标气体、标准气 体 1 和 2 的摩尔浓度测量值; 上标 $\mathrm{L}$ 和 $\mathrm{H}$ 分别为轻和 重同位素。

利用校正后的轻重同位素的摩尔浓度真值直接 计算 $\delta_{\mathrm{a}}$ 值:

$$
\delta_{\mathrm{a}}=\left(\frac{R_{a}}{R_{\mathrm{VPDB}}}-1\right) \times 1000
$$

其中, $R_{a}-{ }^{13} \mathrm{C}=\left[{ }^{13} \mathrm{CO}_{2}\right] /\left[{ }^{12} \mathrm{CO}_{2}\right], R_{a}-{ }^{18} \mathrm{O}=0.5 \times$ $\left[\mathrm{C}^{18} \mathrm{O}^{16} \mathrm{O}\right] /\left[\mathrm{C}^{16} \mathrm{O}_{2}\right]$ 。

少量研究只利用 2 个 $\mathrm{CO}_{2} \delta^{13} \mathrm{C}\left(\right.$ 或 $\left.\delta^{18} \mathrm{O}\right)$ 标准气体 进行浓度依赖性的理论校正, 由于缺乏独立的质控 气体, 无法对数据的校正质量进行评价。例如, Bowling等(2003)利用基于TDLAS技术的TGA100在 美国犹他州灌溉农田生态系统中开展 $\mathrm{CO}_{2} \delta^{13} \mathrm{C}$ 连续 观测研究, 采用 $\mathrm{CO}_{2}$ 为 $363.4 \mu \mathrm{mol} \cdot \mathrm{mol}^{-1}\left(\delta^{13} \mathrm{C}\right.$ 为 $-29.55 \%$ ) 和 $1049.0 \mu \mathrm{mol} \cdot \mathrm{mol}^{-1}\left(\delta^{13} \mathrm{C}\right.$ 为 $-40.58 \%$ ) 2 个标准气体进行浓度依赖性的理论校正。每个测定 循环包括 2 个样品气体和 2 个标准气体, 每个测量 30 
$\mathrm{s}$, 测量循环为 $2 \mathrm{~min}$ 。Pang 等 (2016b) 利用基于 WS-CRDS技术的Picarro G1101-i对北京城市生态系统 大气 $\mathrm{CO}_{2} \delta^{13} \mathrm{C}$ 进行测量, 采用 $\mathrm{CO}_{2}$ 为 $379 \mu \mathrm{mol} \cdot \mathrm{mol}^{-1}$ $\left(\delta^{13} \mathrm{C}\right.$ 为 $\left.-29.36 \%\right)$ 和 $499 \mu \mathrm{mol} \cdot \mathrm{mol}^{-1}\left(\delta^{13} \mathrm{C}\right.$ 为 $\left.-29.95 \%\right)$ 2 个标准气体进行浓度依赖性的理论校正, 每个测 量循环 $320 \mathrm{~min}$ 进行一次校正。Wehr等(2013)利用 QCLAS技术在美国马萨诸塞州哈佛森林生态系统 开展了 ${ }^{12} \mathrm{CO}_{2} 、{ }^{13} \mathrm{CO}_{2}$ 和 $\mathrm{C}^{18} \mathrm{O}^{16} \mathrm{O}$ 的4 $\mathrm{Hz}$ 高频连续观测研 究, 采用 2 个 $\mathrm{CO}_{2}$ 分别为 $350 \mu \mathrm{mol} \cdot \mathrm{mol}^{-1}$ 和 450 $\mu \mathrm{mol} \cdot \mathrm{mol}^{-1}$ 的标准气体进行浓度依赖性的理论校正, 校正频率为 $40 \mathrm{~min}$ 。

大部分研究利用 3 个或者 3 个以上 $\mathrm{CO}_{2} \delta^{13} \mathrm{C}$ (或 $\delta^{18} \mathrm{O}$ )标准气体进行相互检验, 以探讨不同 $\mathrm{CO}_{2} \delta^{13} \mathrm{C}$ (或 $\delta^{18} \mathrm{O}$ )标准气体校正结果的准确性。例如: Wen等 (2013)利用基于WS-CRDS技术的Picarro G1101-i和 基于OA-ICOS技术的Los Gatos DLT-100在北京进行 城市大气 $\mathrm{CO}_{2} \delta^{13} \mathrm{C}$ 测量, 采用 3 个 $\mathrm{CO}_{2}$ 在 361.25$436.41 \mu \mathrm{mol} \cdot \mathrm{mol}^{-1}$ 和 $\delta^{13} \mathrm{C}$ 在 $-10.134--8.652 \%$ 的标 准气体进行浓度依赖性的理论校正, 每个标准气体 测量 $10 \mathrm{~min}$, 每个测量循环 $30 \mathrm{~min}$ 。利用每个测量循 环的 3 个标准气体进行互相校正并评价校正结果的 准确性, 校正后数据的准确性均优于 $0.03 \%$ 。 Pang 等(2016a)利用基于WS-CRDS技术的Picarro G1101-i 在北京进行城市大气 $\mathrm{CO}_{2} \delta^{13} \mathrm{C}$ 测量, 采用 $\mathrm{CO}_{2}$ 为 $368.1 、 451.7$ 和 $550.1 \mu \mathrm{mol} \cdot \mathrm{mol}^{-1}, \delta^{13} \mathrm{C}$ 均为 $-20.38 \%$ 。 的 3 个标准气体进行浓度依赖性的理论校正, 每个 标准气体测量 $40 \mathrm{~min}$, 每个测量循环 $120 \mathrm{~min}$, 同样 每个测量循环的 3 个标准气体进行互相校正并评价 校正结果的准确性, 校正后G1101-i和G2201-i的准 确性分别为 $-0.04 \%{ }_{0}-0.09 \%$ 和 $-0.13 \% 0-0.03 \%$ 。需要 注意的是, 虽然理论上要求标准气体同位素组成涵 盖或接近待测样品气体同位素组成, 但实践中在浓 度依赖性的理论校正中标准气体同位素组成是否有 差异并涵盖或接近待测样品气体同位素组成不是最 重要的, 需要至少保证标准气体 $\mathrm{CO}_{2}$ 浓度能够涵盖 样品气体的 $\mathrm{CO}_{2}$ 浓度变化范围。标准气体 $\mathrm{CO}_{2}$ 浓度范 围涵盖样品气体 $\mathrm{CO}_{2}$ 浓度时, 校正的准确性更高 (Bowling et al., 2003; Wen et al., 2013)。实际数据校 正中相互检验后也可以将所有 $\mathrm{CO}_{2}$ 标准气体用于数 据校正, 不留专门的质控标准气体, 也可以提高校 正精度。

部分相关研究中也专门设置了 $\mathrm{CO}_{2} \delta^{13} \mathrm{C}$ (或 $\delta^{18} \mathrm{O}$ )质控标准气体, 用于评价校正结果的准确性。 例如: Griffis等(2005)利用TGA100在美国明尼苏达 大学农田生态系统开展大气 $\mathrm{CO}_{2} \delta^{18} \mathrm{O}$ 连续观测研究, 采用 $\mathrm{CO}_{2}$ 为 $348.61 \mu \mathrm{mol} \cdot \mathrm{mol}^{-1}(-2.90 \%)$ 和 568.44 $\mu \mathrm{mol} \cdot \mathrm{mol}^{-1}(-14.89 \%) 2$ 个标准气体进行浓度依赖 性的理论校正。每 $2 \mathrm{~min}$ 的测量循环校正一次。同时, 利用上述 2 个标准气体和 1 个质控标准气体进行连续 $11 \mathrm{~h}$ 的循环测量, 利用 2 个标准气体对质控标准气体 进行浓度依赖性的理论校正, 研究表明 2 min $\mathrm{C}^{16} \mathrm{O}_{2}$ 和 $\mathrm{C}^{18} \mathrm{O}^{16} \mathrm{O}$ 和 $\delta^{18} \mathrm{O}$ 值的测量精度分别为 $0.09 \mu \mathrm{mol}$. $\mathrm{mol}^{-1} 、 0.0004 \mu \mathrm{mol} \cdot \mathrm{mol}^{-1}$ 和 $0.26 \%$, 而 $30 \mathrm{~min}$ 的测 量精度提升到 $0.02 \mu \mathrm{mol} \cdot \mathrm{mol}^{-1} 、 0.0001 \mu \mathrm{mol} \cdot \mathrm{mol}^{-1}$ 和 $0.07 \%$ 。Bowling等(2005)利用TGA100在美国科罗 拉多州亚高山针叶林生态系统开展大气 $\mathrm{CO}_{2} \delta^{13} \mathrm{C}$ 连 续观测研究, 共用了 6 个 $\mathrm{CO}_{2}$ 在353.3-477.4 $\mu \mathrm{mol}$. $\mathrm{mol}^{-1}$ 和 $\delta^{13} \mathrm{C}$ 在 $-10.02 \% \mathrm{o}-8.17 \%$ 的标准气体, 利用其 中2-4个标准气体进行浓度依赖性的理论校正, 校 正频率为 $6 \mathrm{~min}$ 。研究表明, 3 点或者 4 点线性回归校 正即增加回归方程中标准气体的数量能够降低标准 气体测量值与真值间的残差。Schaeffer等(2008)利用 TGA100对美国AmeriFlux的Niwot Ridge亚高山针叶 林生态系统开展大气 $\mathrm{CO}_{2} \delta^{13} \mathrm{C}$ 连续观测研究, 采用 4 个 $\mathrm{CO}_{2}$ 浓度在 $350-500 \mu \mathrm{mol} \cdot \mathrm{mol}^{-1}$ 和 $\delta^{13} \mathrm{C}$ 在 $-8.5 \%$ $-14.5 \%$ 的标准气体通过拟合二阶多项式曲线进行 浓度依赖性的理论校正, 校正频率为 $10 \mathrm{~min}$ 。研究 表明, 校正后 2 个质控标准气体 $\mathrm{CO}_{2}$ 与真值的差异分 别为 $(0.04 \pm 0.18) \mu \mathrm{mol} \cdot \mathrm{mol}^{-1}$ 和 $(0.02 \pm 0.16) \mu \mathrm{mol} \cdot \mathrm{mol}^{-1}$, $\delta^{13} \mathrm{C}$ 与真值的差异分别为 $-0.17 \% 0 \pm 0.33 \% 0$ 和 $-0.14 \% 0$ $\pm 0.34 \%$ 。

\section{3 浓度依赖性的经验校正方法与应用}

在无法准确获得标准气体 ${ }^{12} \mathrm{CO}_{2}$ 和 ${ }^{13} \mathrm{CO}_{2}$ 或 $\mathrm{C}^{16} \mathrm{O}_{2}$ 和 $\mathrm{C}^{18} \mathrm{O}^{16} \mathrm{O}$ 浓度真值而已知 $\delta^{13} \mathrm{C}$ 或 $\delta^{18} \mathrm{O}$ 真值的 条件下, 可以考虑通过直接建立 $\delta^{13} \mathrm{C}$ 或 $\delta^{18} \mathrm{O}$ 测量值 与 $\mathrm{CO}_{2}$ 测定值关系的多项式拟合经验方程, 对浓度 依赖性进行校正(Tuzson et al., 2008; Guillon et al., 2012; Sturm et al., 2012)。通常可以直接测定具有相 同 $\delta^{13} \mathrm{C}$ (或 $\delta^{18} \mathrm{O}$ ) 的不同浓度的 $\mathrm{CO}_{2}$ 标准气体进而建 立 $\delta$ 测量值与 $\mathrm{CO}_{2}$ 浓度的多项式拟合经验方程; 或者 利用单个已知 $\delta^{13} \mathrm{C}\left(\right.$ 或 $\left.\delta^{18} \mathrm{O}\right)$ 真值的高浓度 $\mathrm{CO}_{2}$ 稀释 产生不同 $\mathrm{CO}_{2}$ 浓度梯度序列, 然后测定具有相同 $\delta^{13} \mathrm{C}\left(\right.$ 或 $\left.\delta^{18} \mathrm{O}\right)$ 的不同浓度的 $\mathrm{CO}_{2}$ 进而建立 $\delta$ 测量值与 $\mathrm{CO}_{2}$ 浓度的多项式拟合经验方程。例如, 建立3次多 
项式经验方程的校正方程可以表示为:

$$
\begin{aligned}
\delta_{\text {std }, M}= & a\left(\mathrm{CO}_{2 \mathrm{std}, M}\right)^{3}+b\left(\mathrm{CO}_{2 \mathrm{std}, M}\right)^{2}+ \\
& c\left(\mathrm{CO}_{2 \mathrm{std}, M}\right)+d
\end{aligned}
$$

其中 $a 、 b 、 c$ 和 $d$ 分别为经验方程拟合系数, 下标中 $\operatorname{std}$ 表示 $\mathrm{CO}_{2}$ 标准气体, $M$ 表示测量值。

通常建立上述多项式经验拟合方程后, 可以直 接利用该方程和 $\delta^{13} \mathrm{C}$ 或 $\delta^{18} \mathrm{O}$ 真值直接进行经验浓度 依赖性校正, 或利用该函数关系将所有测定数据标 准化到某 $\mathrm{CO}_{2}$ 浓度(如 $400 \mu \mathrm{mol} \cdot \mathrm{mol}^{-1}$ ) 进行经验浓度 依赖性校正, 然后再建立该 $\mathrm{CO}_{2}$ 浓度下 $\mathrm{CO}_{2} \delta^{13} \mathrm{C}$ (或 $\delta^{18} \mathrm{O}$ )标准气体的测量值与真值的单点、两点或多点 函数关系并进行 $\delta$ 值校正(Tuzson et al., 2008; Guillon et al., 2012; Sturm et al., 2012)。

例如，Tuzson等(2008)利用QCLAS技术在瑞士 中部苏黎世草地生态系统测定大气 $\mathrm{CO}_{2} \delta^{13} \mathrm{C}$ 和 $\delta^{18} \mathrm{O}$ 。采用 4 个 $\mathrm{CO}_{2}$ 浓度在 $1000-1970 \mu \mathrm{mol} \cdot \mathrm{mol}^{-1}$ 和 $\delta^{13} \mathrm{C}$ 在 $-37 \%$ - $-4 \%$ 的标准气体分别进行稀释实验, 研究表明不同同位素组成的标准气体测试的浓度依 赖性均表现出 $\delta$ 值与 $\mathrm{CO}_{2}$ 浓度的线性相关, 不受标准 气体同位素组成差异的影响。因此在校正过程中 2 个标准气体用于确定浓度依赖性的线性经验方程。 Sturm等(2012)利用QCLAS技术在瑞士Lägeren森林 生态系统进行大气 ${ }^{12} \mathrm{CO}_{2} 、{ }^{13} \mathrm{CO}_{2}$ 和 $\mathrm{CO}^{18} \mathrm{O}$ 通量的连 续原位测量, 利用稀释法产生 $\delta$ 值相同而 $\mathrm{CO}_{2}$ 浓度在 300-500 $\mu \mathrm{mol} \cdot \mathrm{mol}^{-1}$ 的标准气体进行浓度依赖性的 经验方程校正。Wada等(2011)利用QCLAS技术对日 本名古屋城市大气 $\mathrm{CO}_{2} \delta^{13} \mathrm{C}$ 和 $\delta^{18} \mathrm{O}$ 进行测量, 首先 利用 1 个 $\mathrm{CO}_{2}$ 标准气体在实验前通过一次稀释实验 进行仪器浓度依赖性的经验方程校正, 仪器表现出 线性的浓度依赖性, $\mathrm{CO}_{2}$ 浓度每改变 $20 \mu \mathrm{mol} \cdot \mathrm{mol}^{-1}$ 时, 相应的 $\delta^{13} \mathrm{C}$ 变化为 $-4 \%$ 。 Sturm等(2013)利用 QCLAS技术在瑞士阿尔卑斯山少女峰进行大气 $\mathrm{CO}_{2}$ $\delta^{13} \mathrm{C}$ 和 $\delta^{18} \mathrm{O}$ 观测, 每 $30 \mathrm{~min}$ 测定 $\mathrm{CO}_{2} \delta^{13} \mathrm{C}$ 和 $\delta^{18} \mathrm{O}$ 标准 气体 $5 \mathrm{~min}$ 以校正仪器的时间漂移; 随后每 5 天利用 1 个标准气体通过稀释试验进行浓度依赖性的经验 方程校正。

\section{3 时间漂移的校正原理、方法和应用}

\section{1 时间漂移产生的原因}

时间漂移是指IRIS仪器持续测量具有相同 $\delta^{13} \mathrm{C}$ (或 $\delta^{18} \mathrm{O}$ )的同一浓度的 $\mathrm{CO}_{2}$ 标准气体时表现出的 $\delta$ 测
量值随时间的变化特征。仪器运行过程中, 激光频 率和强度的改变、腔室内气体温度和压力波动以及 激光老化等仪器自身原因都会造成 $\delta$ 测量值的时间 漂移(Sturm et al., 2013), 外界温度、湿度等环境参 数变化的影响也会造成 $\delta$ 测量值的时间漂移(Guillon et al., 2015)。通常, 为了提高仪器测量结果的稳定 性, 减小仪器时间漂移的幅度, 仪器制造商会对仪 器的温度、压力和激光等进行精确控制。同时, 在 仪器的使用过程中, 仪器使用者需要尽可能控制仪 器周围的环境参数稳定, 如有可能建议对 $\mathrm{CO}_{2}$ 气体 进行干燥, 以保持样品的水汽浓度维持在较低的水 平(Guillon et al., 2015)。

IRIS仪器对环境条件变化(如温度和压力等)的 敏感性会导致仪器 $\delta$ 测量值的时间漂移, 即同一浓 度和 $\delta$ 值的 $\mathrm{CO}_{2}$ 标准气体在不同时间的测量结果会 发生变化(Schaeffer et al., 2008; Pang et al., 2016a)。 Vogel等(2013)利用 2 个 $\mathrm{CO}_{2} \delta^{13} \mathrm{C}$ 标准气体持续 $48 \mathrm{~h}$ 的 切换测试(分别测量 $10 \mathrm{~min}$ 和 $20 \mathrm{~min}$ ), 发现基于 WS-CRDS技术的Picarro G1101-i在一天内的漂移能 够达到 $0.3 \%$ 。此外, 浓度依赖性特征也会随着时间 发生变化和漂移。Guillon等(2015)研究表明, 基于 OA-ICOS技术的Los Gatos Research CCIA-EP在 $0-12$ 天的不同时间间隔重复进行 $\mathrm{CO}_{2} \delta^{13} \mathrm{C}$ 浓度依赖 性测试，在日尺度上保持着相似的形状，但是会表 现出随机的时间漂移, $24 \mathrm{~h}$ 的漂移可以达到 $5 \%$ 。

\section{2 时间漂移的校正方法与应用}

通过设置不同的仪器校正频率可以有效地校正 仪器的时间漂移。校正频率是综合权衡仪器时间漂 移、最大校正精度和最小校正时间消耗后的最优校 正间隔(Aemisegger et al., 2012; Vogel et al., 2013)。 为了消除仪器时间漂移的影响, 可以通过Allan方差 测试对仪器性能的测试确定适当的校正频率, 也可 以通过设置一定的时间间隔对 $\mathrm{CO}_{2} \delta^{13} \mathrm{C}\left(\right.$ 或 $\delta^{18} \mathrm{O}$ )标 准气体进行动态测量确定适当的校正频率(Bowling et al., 2003; Griffis et al., 2005; Vogel et al., 2013)。

以往研究已经开展对IRIS仪器的稳定性和精度 的各种测试研究, 基于这些仪器稳定性和精度的测 试结果可以确定适当的时间漂移校正频率, 以获得 符合试验精度需求的校正结果。例如, Guillon等 (2012)对基于OA-ICOS技术的Los Gatos Research DLT-100碳同位素分析仪进行Allan方差测试，研究 表明仪器最优精度的平均时间为 $200 \mathrm{~s}, \mathrm{CO}_{2}$ 浓度和 
$\delta^{13} \mathrm{C}$ 精度分别为 $2 \mu \mathrm{mol} \cdot \mathrm{mol}^{-1}$ 和 $0.04 \%$ 。Wen等(2013) 对基于WS-CRDS技术的Picarro G1101-i和基于 OA-ICOS技术的Los Gatos DLT100进行Allan方差测 试, 研究表明 ${ }^{12} \mathrm{CO}_{2}$ 的最优精度分别为 $0.013 \mu \mathrm{mol}$. $\mathrm{mol}^{-1}$ 和 $0.016 \mu \mathrm{mol} \cdot \mathrm{mol}^{-1}$, 平均时间为 $1800 \mathrm{~s}$ 和 $500 \mathrm{~s} ; \delta^{13} \mathrm{C}$ 的最优精度分别为 $0.08 \%$ 和 $0.04 \%$, 平均 时间为 $2000 \mathrm{~s}$ 和 $1000 \mathrm{~s}$ 。Vogel等(2013)对基于 WS-CRDS技术的Picarro G1101-i进行Allan方差测 试, 研究表明 $60 \mathrm{~min}$ 平均时间的测量精度能够达到 $0.1 \%$ 。同时, 利用 2 个 $\mathrm{CO}_{2} \delta^{13} \mathrm{C}$ 标准气体分别测量 $10 \mathrm{~min}$ 和 $20 \mathrm{~min}$ 的切换测试, 表明 2 个标准气体的漂 移状况相似, 在一天内约为 $0.3 \%$ 。 Pang等(2016a)对 基于WS-CRDS技术的G1101-i和G2201-i的Allan方 差测试, 研究表明 $\mathrm{G} 1101-\mathrm{i}$ 和 $\mathrm{G} 2201-\mathrm{i}$ 的 $\delta^{13} \mathrm{C}$ 最优精 度分别为 $0.08 \% 0-0.15 \%$ 和 $0.01 \% 0-0.04 \%$, 平均时间 为 $1900-7600 \mathrm{~s}$ 和 $3800-7600 \mathrm{~s}$ 。

前面阐述的浓度依赖性的理论校正和经验校正 均指某个时刻的浓度依赖性校正而未涉及时间漂移 问题。通常可以直接设置固定的校正频率, 定期测 量 $\mathrm{CO}_{2}$ 标准气体进行浓度依赖性的理论或经验动态 校正, 这样在浓度依赖性校正的同时也对仪器的时 间漂移进行了校正(Bowling et al., 2003, 2005; Griffis et al., 2005; Schaeffer et al., 2008; Pang et al., 2016b)。还有研究者为了提高时间漂移校正的准确 性, 定期测量 $\mathrm{CO}_{2}$ 标准气体的 $\mathrm{CO}_{2}$ 浓度和 $\delta^{13} \mathrm{C}$, 利用 相邻两个时间的测量结果分别拟合标准参考气体 $\mathrm{CO}_{2}$ 浓度和 $\delta^{13} \mathrm{C}$ 随时间变化的线性回归方程, 通过 时间线性内插以获得所需更高校正频率的 $\mathrm{CO}_{2}$ 浓度 和 $\delta^{13} \mathrm{C}\left(\right.$ 或 $\delta^{18} \mathrm{O}$ ) 时间序列数据, 利用内插后生成的 标准气体数据对样品测量结果进行校正, 从而进一 步减少时间漂移的校正误差(Wehr et al., 2013; Guillon et al., 2015)。为了减少校正误差, 在校正时 也利用平滑样条对每个测量循环的校正系数进行平 滑处理, 然后应用平滑后的校正系数对数据进行校 正(Sturm et al., 2012, 2013)。

也有研究者单独设置 $\mathrm{CO}_{2}$ 标准气体对仪器的时 间漂移进行校正(Tuzson et al., 2008; Sturm et al., 2012, 2013)。例如, Tuzson等(2008)利用QCLAS技术 在瑞士中部苏黎世草地生态系统测定大气 $\mathrm{CO}_{2} \delta^{13} \mathrm{C}$ 和 $\delta^{18} \mathrm{O}$ 。校正过程中设置 3 个标准气体, 2 个用于确定 浓度依赖性的线性经验方程, 另外 1 个用于时间漂 移校正, 校正频率为 $30 \mathrm{~min}$ 。在整个观测时期内校
正后 $\delta^{13} \mathrm{C}$ 和 $\delta^{18} \mathrm{O}$ 的精度和准确性都在 $0.2 \%$ 左右。 Wada等(2011)利用QCLAS技术对日本名古屋城市 大气 $\mathrm{CO}_{2} \delta^{13} \mathrm{C}$ 和 $\delta^{18} \mathrm{O}$ 进行测量, 首先进行仪器浓度 依赖性的经验方程校正，随后每30 min采用 2 个参 考气体 $\left(\mathrm{CO}_{2}=498.34 \mu \mathrm{mol} \cdot \mathrm{mol}^{-1}, \delta^{13} \mathrm{C}=-12.68 \%\right.$, $\delta^{18} \mathrm{O}=-9.71 \% ; \mathrm{CO}_{2}=413.28 \mu \mathrm{mol} \cdot \mathrm{mol}^{-1}, \delta^{13} \mathrm{C}=$ $-9.11 \%$; $\delta^{18} \mathrm{O}=-7.07 \%$ ) 进行两点时间漂移校正。 Sturm等(2012)利用QCLAS技术在瑞士Lägeren森林 生态系统进行大气 ${ }^{12} \mathrm{CO}_{2} 、{ }^{13} \mathrm{CO}_{2}$ 和 $\mathrm{CO}^{18} \mathrm{O}$ 通量的连 续原位测量, 利用浓度依赖性的经验方程校正, 然 后利用 2 个已知 $\mathrm{CO}_{2}$ 浓度和 $\delta^{13} \mathrm{C}\left(\right.$ 或 $\delta^{18} \mathrm{O}$ ) 的校正气体 进行两点时间漂移校正。校正频率为 $1 \mathrm{~h}$, 其中 $5 \mathrm{~min}$ 测量校正气体, $55 \mathrm{~min}$ 测量样品气体。5 min的校正 气体测量包括稀释校正 $120 \mathrm{~s}$, 以及 2 个标准气体和 1 个质控气体分别测量 $60 \mathrm{~s}$ 。在连续3 周的野外观测中, 每 $1 \mathrm{~h}$ 一次校正后, $\delta^{13} \mathrm{C}$ 和 $\delta^{18} \mathrm{O}$ 测量结果的精度为 $0.15 \%$ 。Sturm等(2013)利用QCLAS技术在瑞士阿尔 卑斯山少女峰进行大气 $\mathrm{CO}_{2} \quad \delta^{13} \mathrm{C}$ 和 $\delta^{18} \mathrm{O}$ 观测, 每隔 $30 \min$ 测定 $\mathrm{CO}_{2} \delta^{13} \mathrm{C}$ 和 $\delta^{18} \mathrm{O}$ 标准气体 $5 \mathrm{~min}$ 以校正仪 器的时间漂移; 随后每 5 天利用 1 个标准气体通过稀 释试验进行浓度依赖性的经验方程校正。在平均时 间为 $10 \mathrm{~min}$ 时, $\delta^{13} \mathrm{C}$ 和 $\delta^{18} \mathrm{O}$ 的测量精度均可以达到 $0.02 \%$, 准确性约为 $0.1 \%$ 。

\section{4 数据溯源的校正原理、方法和应用}

IRMS 是相对测量技术, 而IRIS 是绝对测量技 术。所有的IRMS和IRIS仪器测定的 $\delta$ 值均需要利用 1 个或者多个标准物质溯源到同位素国际标准, 这一 过程称为数据溯源。IRIS技术实现了对空气 $\mathrm{CO}_{2}$ $\delta^{13} \mathrm{C}$ 和 $\delta^{18} \mathrm{O}$ 的连续测定, 但需要IRMS提供数据溯源 至 $\mathrm{CO}_{2}$ 碳或氧同位素国际标准(Vienna Pee Dee Belemnite)的 $\mathrm{CO}_{2} \delta^{13} \mathrm{C}$ 和 $\delta^{18} \mathrm{O}$ 标准气体(Wen et al., 2013; Pang et al., 2016a; Wang et al., 2017)。

数据溯源方法通常包括单点溯源和两点或多点 溯源:

(1) 单点溯源: 利用单个已知 $\delta$ 值的标准物质 $\left(\delta_{\mathrm{std}, \mathrm{T}}\right)$ 溯源, 只适用于标准物质与样品 $\delta$ 值较为接近 的情况。样品的真值 $\delta_{\mathrm{s}, \mathrm{T}}$ 为:

$$
\delta_{\mathrm{s}, \mathrm{T}}=\delta_{\mathrm{s}, \mathrm{M}}+\left(\delta_{\mathrm{std}, \mathrm{T}}-\delta_{\mathrm{std}, \mathrm{M}}\right)
$$

式中下标 $\mathrm{T}$ 和 $\mathrm{M}$ 分别为真值和测定值, $\mathrm{s}$ 和 $\mathrm{std}$ 分别为 样品和标准物质。

(2)两点或多点溯源: 建立两个或多个涵盖样品 
$\delta$ 值的标准物质真值 $\left(\delta_{\mathrm{std}, \mathrm{T}}\right)$ 和测定值 $\left(\delta_{\mathrm{std}, \mathrm{M}}\right)$ 的线性关 系, 获得线性回归拟合参数并用于对样品进行数据 溯源, 样品的真值 $\delta_{\mathrm{T}, \mathrm{s}}$ 为:

$$
\delta_{\mathrm{s}, \mathrm{T}}=a \times \delta_{\mathrm{s}, \mathrm{M}}+b
$$

其中, $a$ 和 $b$ 分别为线性回归方程的拟合参数。

在前面阐述的浓度依赖性和时间漂移的校正中, 如果采用的已知 $\mathrm{CO}_{2}$ 浓度和 $\delta^{13} \mathrm{C}\left(\right.$ 或 $\left.\delta^{18} \mathrm{O}\right)$ 值的标准 气体已经溯源国际标准, 则在浓度依赖性和时间漂 移校正的同时就实现了数据溯源(Bowling et al., 2003, 2005; Griffis et al., 2005; Schaeffer et al., 2008; Wen et al., 2013; Pang et al., 2016a, 2016b)。如果上 述浓度依赖性和时间漂移校正 $\mathrm{CO}_{2}$ 标准气体未溯源 至国际标准, 则需要利用已知 $\delta^{13} \mathrm{C}\left(\right.$ 或 $\delta^{18} \mathrm{O}$ ) 值的校 正气体进行单点、两点或者多点数据溯源(Tuzson et al., 2008; Wada et al., 2011; Sturm et al., 2012, 2013)。例如, Sturm等(2013)利用QCLAS技术在瑞士 阿尔卑斯山少女峰进行大气 $\mathrm{CO}_{2} \delta^{13} \mathrm{C}$ 和 $\delta^{18} \mathrm{O}$ 观测, 每 6 h 测量 3 个标准气体, 其中 2 个进行数据溯源, 另 1 个用于检查校正的稳定性。

\section{5 研究展望}

\section{1 加强不同IRIS仪器和校正方法的比对和一致 性研究}

由于IRIS仪器直接测量每个核素的浓度, 单个 核素浓度值的测定误差导致了IRIS仪器的浓度依赖 性(Griffith et al., 2012)。此外, 由于仪器的时间漂移, 需要设置一定的校正频率进行定期校正以消除仪器 漂移的影响。以往研究主要集中在单台IRIS仪器的 方法论研究方面(Bowling et al., 2003; Tuzson et al., 2008; Griffith et al., 2012; Guillon et al., 2012; Vogel et $a l ., 2013)$ 。较少关注IRIS仪器之间和不同校正方法之 间的对比研究(Griffis, 2013; Wen et al., 2013), 仅有 Wen等(2013)对两个IRIS技术的 $\mathrm{CO}_{2} \delta^{13} \mathrm{C}$ 分析仪的 对比研究表明Los Gatos DLT-100和Picarro G1101-i 对大气 $\mathrm{CO}_{2} \delta^{13} \mathrm{C}$ 测量结果标定后的一致性在 $-0.02 \%$ 。 $\pm 0.18 \%$ 以内, 然而这种微小的差异由于存在一定 的浓度依赖性会导致Keeling Plot的分析误差达到 $2.44 \%$ 。不同的IRIS仪器设备之间测量结果如果存 在系统偏差, 会导致不同仪器测量结果不具有可比 性(Flowers et al., 2012; Hammer et al., 2013; Griffis, 2013; Wen et al., 2013)。在今后的研究中, 需要重点 关注不同IRIS仪器和不同校正方法间的对比研究,
加强数据质量控制, 确保不同测量结果间的准确 性、一致性和可比性。推荐至少利用 3 个或 3 个以上 已知 $\mathrm{CO}_{2}$ 浓度和 $\delta$ 真值的标准气体涵盖待测 $\mathrm{CO}_{2}$ 浓度 校正浓度依赖性，设置适当的校正频率校正时间漂 移并溯源至国际标准。目前, IRIS技术的测量结果经 过浓度依赖性和时间漂移校正后准确性能够达到 $0.01 \%$ o $\pm 0.03 \%$ (Bowling et al., 2005), 与IRMS相当。

\section{$5.2 \mathrm{H}_{2} \mathrm{O} 、 \mathrm{CH}_{4}$ 和 $\mathrm{N}_{2} \mathrm{O}$ 等IRIS技术面临同样的校正 难题}

随着IRIS技术的发展, 除了 $\mathrm{CO}_{2}$ 外, 也可以实 现 $\mathrm{H}_{2} \mathrm{O} 、 \mathrm{CH}_{4}$ 和 $\mathrm{N}_{2} \mathrm{O}$ 等多种同位素气体的原位连续测 量, 在这些测量中同样需要考虑仪器的浓度依赖性 和时间漂移等误差来源, 并通过适当的校正策略获 得精度和准确度均满足研究需要的测量结果(Wen et al., 2008, 2012; Keppler et al., 2010; Mohn et al., 2012, 2014; Yamamoto et al., 2014), 例如: Sturm和 Knohl (2010)发现基于 OA-ICOS技术的Los Gatos DLT-100在 $2000-27000 \mu \mathrm{mol} \cdot \mathrm{mol}^{-1} \mathrm{H}_{2} \mathrm{O}$ 浓度范围 内氛同位素组成 $(\delta \mathrm{D})$ 和 $\delta^{18} \mathrm{O}$ 测量值存在着明显的浓 度依赖性。Aemisegger等(2012)发现基于WS-CRDS 技术的Picarro L1115-i测量中, 由于气体样品中水 汽浓度的变化导致的 $\delta \mathrm{D}$ 和 $\delta^{18} \mathrm{O}$ 的偏差能够分别达 到4\%o和 $2 \%$ 。 Y Yamamoto等(2014)发现基于QCLAS 技术在1 $000-2500 \mathrm{nmol} \cdot \mathrm{mol}^{-1}$ 的 $\mathrm{N}_{2} \mathrm{O}$ 浓度范围内 $\delta^{15} \mathrm{~N}_{\alpha}$ 和 $\delta^{15} \mathrm{~N}_{\beta}$ 存在明显的浓度依赖性。这些浓度依赖 性可以通过理论校正或者经验方程校正予以消除 (Wen et al., 2008; Aemisegger et al., 2012)。Kurita等 (2012)发现基于OA-ICOS技术的Los Gatos DLT-100 连续 4 个月重复测量水汽标准 $\delta \mathrm{D}$ 和 $\delta^{18} \mathrm{O}$ 的漂移范围 分别在 $-77 \% 0--82 \%$ 和 $-8.0 \% 0--12.5 \%$ 之间。Keppler 等(2010)发现基于OA-ICOS技术在连续 6 天的试验 期间 $\delta^{13} \mathrm{C}_{\mathrm{CH} 4}$ 的漂移达到 $0.84 \%$ 。利用与 $\mathrm{CO}_{2}$ 相似的 校正方法, 可以对 $\mathrm{H}_{2} \mathrm{O} 、 \mathrm{CH}_{4}$ 和 $\mathrm{N}_{2} \mathrm{O}$ 等多种同位素测 量结果进行浓度依赖性和时间漂移校正, 以获得可 追溯到国际标准的原位连续测量数据, 为在不同时 空尺度上研究植被-大气碳水交换的生物物理过程、 甲烷生产/消耗、硝化/反硝化作用的环境生物控制 机制提供新的机遇。

\subsection{IRIS技术在生态学中的应用潜力}

IRIS技术的发展带来的 $\mathrm{CO}_{2}$ 碳氧同位素的原位 连续观测极大地拓展了我们对生态系统、区域和大 陆尺度影响碳收支的生物物理过程的研究和解释。 
IRIS技术与浴度协方差、通量廓线和动态箱式技术 相结合可以实现 $\mathrm{CO}_{2}$ 碳氧同位素比值和通量的原位 连续观测(Griffis et al., 2004, 2005; Zhang et al., 2006; Santos et al., 2012), 有助于我们深入理解生 态系统碳周转、分配和利用等一系列复杂的生物物 理过程及其环境和生物控制机制。例如, Wingate等 (2010)利用TDLAS技术与通量廓线和动态箱式技术 结合, 研究表明茎秆、土壤和生态系统呼吸的 $\mathrm{CO}_{2}$ $\delta^{13} \mathrm{C}$ 比光合 $\delta^{13} \mathrm{C}$ 分馏存在更小的短期变异并存在 2-10天的滞后效应。Wehr等(2016)利用QCLAS技术 与涡度协方差技术结合进行通量拆分, 研究表明传 统拆分方法高估了生长季前期的生态系统光合和白 天呼吸, 错误地描述了生态系统光能利用效率。在 全球通量网络(Fluxnet)中, 同位素观测相对较少且 多限制在短期集中观测。随着IRIS技术的发展, 未 来在更多的通量观测站点结合进行碳氧同位素的野 外连续原位观测, 将有助于提升我们对生态系统和 大气间碳水交换生物物理过程的理解 (Griffis, 2013)。此外, 通过野外同位素标记示踪实验结合原 位连续观测，可以有效地评价和验证同位素生态系 统模型(例如SiLSM和CLM-CN 3.5)中的光合作用、 呼吸、碳库和分配过程与机制等。

\section{参考文献}

Aemisegger F, Sturm P, Graf P, Sodemann H, Pfahl S, Knohl A, Wernli $\mathrm{H}$ (2012). Measuring variations of $\delta^{18} \mathrm{O}$ and $\delta \mathrm{D}$ in atmospheric water vapour using two commercial laser-based spectrometers: An instrument characterisation study. Atmospheric Measurement Techniques, 5, 1491-1511.

Bickford CP, Hanson DT, McDowell NG (2010). Influence of diurnal variation in mesophyll conductance on modelled ${ }^{13} \mathrm{C}$ discrimination: Results from a field study. Journal of Experimental Botany, 61, 3223-3233.

Bowling DR, Burns SP, Conway TJ, Monson RK, White JWC (2005). Extensive observations of $\mathrm{CO}_{2}$ carbon isotope content in and above a high-elevation subalpine forest. Global Biogeochemical Cycles, 19, GB3023. DOI: 10.1029/2004GB002394.

Bowling DR, Sargent SD, Tanner BD, Ehleringer JR (2003). Tunable diode laser absorption spectroscopy for stable isotope studies of ecosystem-atmosphere $\mathrm{CO}_{2}$ exchange. Agricultural and Forest Meteorology, 118, 1-19.

Chen CH, Pang JP, Wei J, Wen XF, Sun XM (2017). Inter-comparison among three models for the $\delta^{13} \mathrm{C}$ of respiration with four regression approaches. Agricultural and Forest Meteorology, 247, 229-239

Flowers BA, Powers HH, Dubey MK, McDowell NG (2012).
Inter-comparison of two high-accuracy fast-response spectroscopic sensors of carbon dioxide: A case study. Atmospheric Measurement Techniques, 5, 991-997.

Griffis TJ (2013). Tracing the flow of carbon dioxide and water vapor between the biosphere and atmosphere: A review of optical isotope techniques and their application. Agricultural and Forest Meteorology, 174, 85-109.

Griffis TJ, Baker JM, Sargent SD, Tanner BD, Zhang J (2004). Measuring field-scale isotopic $\mathrm{CO}_{2}$ fluxes with tunable diode laser absorption spectroscopy and micrometeorological techniques. Agricultural and Forest Meteorology, 124, 15-29.

Griffis TJ, Lee X, Baker JM, Sargent SD, King JY (2005). Feasibility of quantifying ecosystem-atmosphere $\mathrm{C}^{18} \mathrm{O}^{16} \mathrm{O}$ exchange using laser spectroscopy and the flux-gradient method. Agricultural and Forest Meteorology, 135, 44-60.

Griffith DWT, Deutscher NM, Caldow C, Kettlewell G, Riggenbach M, Hammer S (2012). A fourier transform infrared trace gas and isotope analyser for atmospheric applications. Atmospheric Measurement Techniques, 5, 2481-2498.

Guillon S, Agrinier P, Pili E (2015). Monitoring $\mathrm{CO}_{2}$ concentration and $\delta^{13} \mathrm{C}$ in an underground cavity using a commercial isotope ratio infrared spectrometer. Applied Physics B-Lasers and Optics, 119, 165-175.

Guillon S, Pili E, Agrinier P (2012). Using a laser-based $\mathrm{CO}_{2}$ carbon isotope analyser to investigate gas transfer in geological media. Applied Physics B - Lasers and Optics, 107, 449-457.

Hammer S, Griffith DWT, Konrad G, Vardagl S, Caldow C, Levin I (2013). Assessment of a multi-species in situ ftir for precise atmospheric greenhouse gas observations. Atmospheric Measurement Techniques, 6, 1153-1170.

Keppler F, Laukenmann S, Rinne J, Heuwinkel H, Greule M, Whiticar M, Lelieveld J (2010). Measurements of ${ }^{13} \mathrm{C} /{ }^{12} \mathrm{C}$ methane from anaerobic digesters: Comparison of optical spectrometry with continuous-flow isotope ratio mass spectrometry. Environmental Science \& Technology, 44, 5067-5073.

Kurita N, Newman BD, Araguas-Araguas LJ, Aggarwal P (2012). Evaluation of continuous water vapor $\delta \mathrm{D}$ and $\delta^{18} \mathrm{O}$ measurements by off-axis integrated cavity output spectroscopy. Atmospheric Measurement Techniques, 5, 2069-2080.

Lin GH (2013). Stable Isotope Ecology. Higher Education Press, Beijing. 30. [林光辉 (2013). 稳定同位素生态学. 高等教育出版社，北京. 30.]

McManus JB, Nelson DD, Zahniser MS (2010). Long-term continuous sampling of ${ }^{12} \mathrm{CO}_{2},{ }^{13} \mathrm{CO}_{2}$ and ${ }^{12} \mathrm{C}^{18} \mathrm{O}^{16} \mathrm{O}$ in ambient air with a quantum cascade laser spectrometer. Isotopes in Environmental and Health Studies, 46, 49-63.

Mohn J, Tuzson B, Manninen A, Yoshida N, Toyoda S, Brand WA, Emmenegger L (2012). Site selective real-time measurements of atmospheric $\mathrm{N}_{2} \mathrm{O}$ isotopomers by laser spectroscopy. Atmospheric Measurement Techniques, 5, 1601-1609.

Mohn J, Wolf B, Toyoda S, Lin CT, Liang MC, Bruggemann 
N, Wissel H, Steiker AE, Dyckmans J, Szwec L, Ostrom NE, Casciotti KL, Forbes M, Giesemann A, Well R, Doucett RR, Yarnes CT, Ridley AR, Kaiser J, Yoshida N (2014). Interlaboratory assessment of nitrous oxide isotopomer analysis by isotope ratio mass spectrometry and laser spectroscopy: Current status and perspectives. Rapid Communications in Mass Spectrometry, 28, 1995-2007.

Pang JP, Wen XF, Sun XM, Huang K (2016a). Intercomparison of two cavity ring-down spectroscopy analyzers for atmospheric ${ }^{13} \mathrm{CO}_{2} /{ }^{12} \mathrm{CO}_{2}$ measurement. Atmospheric Measurement Techniques, 9, 3879-3891.

Pang J, Wen X, Sun X (2016b). Mixing ratio and carbon isotopic composition investigation of atmospheric $\mathrm{CO}_{2}$ in Beijing, China. Science of the Total Environment, 539, 322-330.

Santos E, Wagner-Riddle C, Lee X, Warland J, Brown S, Staebler R, Bartlett P, Kim K (2012). Use of the isotope flux ratio approach to investigate the ${ }^{12} \mathrm{C}^{18} \mathrm{O}^{16} \mathrm{O}$ and ${ }^{13} \mathrm{CO}_{2}$ exchange near the floor of a temperate deciduous forest. Biogeosciences, 9, 2385-2399.

Schaeffer SM, Miller JB, Vaughn BH, White JWC, Bowling DR (2008). Long-term field performance of a tunable diode laser absorption spectrometer for analysis of carbon isotopes of $\mathrm{CO}_{2}$ in forest air. Atmospheric Chemistry and Physics, 8, 5263-5277.

Shim JH, Powers HH, Meyer CW, Pockman WT, McDowell N (2011). The role of interannual, seasonal, and synoptic climate on the carbon isotope ratio of ecosystem respiration at a semiarid woodland. Global Change Biology, 17, 2584-2600.

Sturm P, Eugster W, Knohl A (2012). Eddy covariance measurements of $\mathrm{CO}_{2}$ isotopologues with a quantum cascade laser absorption spectrometer. Agricultural and Forest Meteorology, 152, 73-82.

Sturm P, Knohl A (2010). Water vapor $\delta^{2} \mathrm{H}$ and $\delta^{18} \mathrm{O}$ measurements using off-axis integrated cavity output spectroscopy. Atmospheric Measurement Techniques, 3, 67-77.

Sturm P, Tuzson B, Henne S, Emmenegger L (2013). Tracking isotopic signatures of $\mathrm{CO}_{2}$ at the high altitude site jungfraujoch with laser spectroscopy: Analytical improvements and representative results. Atmospheric Measurement Techniques, 6, 1659-1671.

Tanaka K, Kojima R, Takahashi K, Tonokura K (2013). Continuous measurements of stable carbon isotopes in $\mathrm{CO}_{2}$ with a near-IR laser absorption spectrometer. Infrared Physics \& Technology, 60, 281-287.

Tuzson B, Mohn J, Zeeman MJ, Werner RA, Eugster W, Zahniser MS, Nelson DD, McManus JB, Emmenegger L (2008). High precision and continuous field measurements of $\delta^{13} \mathrm{C}$ and $\delta^{18} \mathrm{O}$ in carbon dioxide with a cryogen-free qclas. Applied Physics B-Lasers and Optics, 92, 451-458.

Vogel FR, Huang L, Ernst D, Giroux L, Racki S, Worthy DEJ (2013). Evaluation of a cavity ring-down spectrometer for

www.plant-ecology.com in situ observations of ${ }^{13} \mathrm{CO}_{2}$. Atmospheric Measurement Techniques, 6, 301-308.

Wada R, Pearce JK, Nakayama T, Matsumi Y, Hiyama T, Inoue G, Shibata T (2011). Observation of carbon and oxygen isotopic compositions of $\mathrm{CO}_{2}$ at an urban site in nagoya using mid-IR laser absorption spectroscopy. Atmospheric Environment, 45, 1168-1174.

Wang J, Wen XF, Li SG (2017). Differentiated correction on the signal intensity dependence of GasBench II-IRMS from blank effect and instrument nonlinear effect. International Journal of Mass Spectrometry, 422, 80-87.

Wehr R, Munger JW, McManus JB, Nelson DD, Zahniser MS, Davidson EA, Wofsy SC, Saleska SR (2016). Seasonality of temperate forest photosynthesis and daytime respiration. Nature, 534, 680-686.

Wehr R, Munger JW, Nelson DD, McManus JB, Zahniser MS, Wofsy SC, Saleska SR (2013). Long-term eddy covariance measurements of the isotopic composition of the ecosystematmosphere exchange of $\mathrm{CO}_{2}$ in a temperate forest. Agricultural and Forest Meteorology, 181, 69-84.

Wen XF, Lee X, Sun XM, Wang JL, Tang YK, Li SG, Yu GR (2012). Inter-comparison of four commercial analyzers for water vapor isotope measurement. Journal of Atmospheric and Oceanic Technology, 29, 235-247.

Wen XF, Meng Y, Zhang XY, Sun XM, Lee X (2013). Evaluating calibration strategies for isotope ratio infrared spectroscopy for atmospheric ${ }^{13} \mathrm{CO}_{2} /{ }^{12} \mathrm{CO}_{2}$ measurement. Atmospheric Measurement Techniques, 6, 1491-1501.

Wen XF, Sun XM, Zhang SC, Yu GR, Sargent SD, Lee X (2008). Continuous measurement of water vapor $\mathrm{D} / \mathrm{H}$ and ${ }^{18} \mathrm{O} /{ }^{16} \mathrm{O}$ isotope ratios in the atmosphere. Journal of $\mathrm{Hy}$ drology, 349, 489-500.

Wingate L, Ogee J, Burlett R, Bosc A, Devaux M, Grace J, Loustau D, Gessler A (2010). Photosynthetic carbon isotope discrimination and its relationship to the carbon isotope signals of stem, soil and ecosystem respiration. New Phytologist, 188, 576-589.

Yakir D, Sternberg LDL (2000). The use of stable isotopes to study ecosystem gas exchange. Oecologia, 123, 297-311.

Yamamoto A, Uchida Y, Akiyama H, Nakajima Y (2014). Continuous and unattended measurements of the site preference of nitrous oxide emitted from an agricultural soil using quantum cascade laser spectrometry with intercomparison with isotope ratio mass spectrometry. Rapid Communications in Mass Spectrometry, 28, 1444-1452.

Zhang J, Griffis TJ, Baker JM (2006). Using continuous stable isotope measurements to partition net ecosystem $\mathrm{CO}_{2}$ exchange. Plant, Cell \& Environment, 29, 483-496. 